(2) Open Access Full Text Article

REVIEW

\title{
Inverted Internal Limiting Membrane Flap Technique: Is It the Best Option for Macular Holes?
}

\author{
Nisa Silva (D) \\ André Ferreira $\mathbb{D D}^{1,2}$ \\ Zofia Anna Nawrocka \\ (vel Michalewska) $\mathbb{D D}^{3}$ \\ Angelina Meireles (D) ${ }^{1,4}$ \\ 'Ophthalmology Department, Centro \\ Hospitalar Universitário do Porto, Porto, \\ 4099-00 I, Portugal; ' Unit of Anatomy, \\ Department of Biomedicine, Faculty of \\ Medicine of University of Porto, Porto, \\ 4200-319, Portugal; ${ }^{3}$ Ophthalmic Clinic \\ Jasne Blonia, Łódź, 9I-I34, Poland; \\ ${ }^{4}$ Department of Ophthalmology, Instituto \\ de Ciências Biomédicas Abel Salazar, \\ University of Porto, Porto, 4050-313 \\ Portugal
}

\begin{abstract}
Surgical treatment is generally necessary to repair full-thickness macular holes (FTMH). Although vitrectomy with or without internal limiting membrane (ILM) peeling remains the standard surgical technique, the inverted ILM flap procedure has increasingly assumed a role in the primary surgical repair of FTMHs. Some vitreoretinal surgeons reserve this technique to treat large or myopic holes, whereas others use it routinely in all cases. This paper is a comprehensive review of the current scientific evidence on the anatomical and functional outcomes of the inverted ILM flap technique in the repair of macular holes, following the International Vitreomacular Traction Study (IVTS) group classification.
\end{abstract}

Keywords: macular hole, inverted internal-limiting membrane flap technique, IVTS classification

\section{Introduction}

The macular hole is a vitreoretinal interface disorder that has evolved in terms of classification and surgical treatment.

\section{Classification Systems}

Currently, there are three main classification systems (by chronological order): Gass classification, ${ }^{1}$ International Vitreomacular Traction Study (IVTS) group classification, ${ }^{2}$ and European Eye Epidemiology (E3) consortium classification. ${ }^{3}$ Distinct from the clinical classification of Gass, the IVTS and E3 classifications are both based on morphologic data extracted from optical coherence tomography (OCT). OCT imaging is widely available in ophthalmology practice, which led to the adoption of OCT-based classification systems for the diagnosis and management of macular holes. Comparing with IVTS classification, OCT-based E3 classification is a more complex staging system for a wide range of macular diseases. The E3 classification has assumed the definition and size subclassification of the macular holes proposed by the IVTS group.

According to OCT-based classifications, a full-thickness macular hole (FTMH) is an anatomic defect located in the fovea involving all retinal layers from the internal limiting membrane (ILM) to the retinal pigment epithelium (RPE). IVTS group subclassified FTMHs by size $(\leq 250 \mu \mathrm{m},>250 \mu \mathrm{m}$ and $\leq 400 \mu \mathrm{m},>400 \mu \mathrm{m})$, vitreous status (presence or absence of vitreomacular traction (VMT)), and cause (primary and secondary).
Correspondence: Nisa Silva

Ophthalmology Department, Centro

Hospitalar Universitário do Porto, Largo do Prof. Abel Salazar, Porto, 4099-00I, Portugal

Email nisapinhosilva@gmail.com 


\section{Surgical Treatment with Inverted ILM Flap Technique and Other Techniques}

Surgical treatment is generally necessary to repair FTMHs. Gass et al first described the pathogenesis and classification of idiopathic macular holes. ${ }^{4}$ In another work, they recognized the importance of removing anterior-posterior and tangentially oriented traction caused by shrinkage of the prefoveal cortical vitreous to prevent idiopathic macular hole formation. ${ }^{5}$ FTMHs were considered an untreatable condition until Kelly and Wendel introduce pars plana vitrectomy with gas tamponade. ${ }^{6}$ Afterwards, the initial procedure has been modified to more advanced techniques, such as ILM peeling, ${ }^{7}$ inverted ILM flap technique, ${ }^{8}$ ILM abrasion, ${ }^{9}$ ILM or neurosensory retina autologous graft, ${ }^{10,11}$ human amniotic membrane graft, ${ }^{12}$ and posterior lens capsule transplantation. ${ }^{13}$ Pharmacologic vitreolysis without surgery, particularly intravitreal injection (IVI) of ocriplasmin, was also attempted in selected cases. ${ }^{14}$

Although vitrectomy with or without ILM peeling remains the standard surgical technique, the inverted ILM flap procedure has increasingly assumed a role in the primary surgical repair of FTMHs. Some vitreoretinal surgeons reserve this technique to treat large or myopic holes, whereas others use it routinely in all cases. ${ }^{15,16}$ Theoretically, when the ILM fills the MH, it acts as a scaffold for the activation, proliferation, and migration of Müller cells, and, consequently, the production of neurotrophic and basic fibroblast growth factors during this process will contribute to hole closure. ${ }^{17}$ When there is no closure of the hole edges, the ILM flap enables "flap closure" in macular holes that otherwise would remain open with bare retinal pigment epithelium after standard ILM peeling. ${ }^{18}$

The surgical steps of classic inverted ILM flap technique are core vitrectomy, membranes staining (eg trypan blue, indocyanine green, brilliant blue G), epiretinal membrane (ERM) peeling (if present), ILM peeling in a circular fashion for approximately 2 disc diameters around the hole, coverage of the hole with an inverted ILM flap, and air or sulfur hexafluoride (SF6) or perfluoropropane (C3F8) gas tamponade. ${ }^{8}$ Face-down positioning is recommended for 5 to 7 days after surgery. In the meantime, modifications to the original technique have been described. ${ }^{19-21}$ Nawrocka (vel Michalewska) et al introduced the novel technique of peeling off solely the ILM temporal to the fovea to minimize the area of surgical trauma. ${ }^{19} \mathrm{Hu}$ et al described a procedure in which a tongue-shaped superior ILM flap is created, while the inferior ILM is not peeled off. ${ }^{21}$ Decreasing the area of ILM peeling has the advantage of inducing fewer inner retinal dimplings and fewer changes in central retinal sensitivity, and macular microvasculature. ${ }^{22-24}$ Aurora et al proposed a technique called cabbage leaf inverted flap ILM peeling in which multiple ILM flaps are inverted over each other like cabbage leaves. ${ }^{25}$ However, there is evidence supporting the use of single-layered flap covering the FTMH instead of multilayered flap or "insertion" technique. ${ }^{26}$ The comparison of "cover" and "insertion" techniques showed that covering the FTMH with ILM flap yielded better visual outcome and recovery of photoreceptor layers, probably due to less iatrogenic trauma to the outer retina. ${ }^{26-29}$ Even in the presence of a retinal detachment (RD), the "cover" technique, as performed in the classic inverted ILM flap technique, is the preferable procedure. ${ }^{30}$ Moreover, the inverted ILM flap technique has shown to be safe with few perioperative complications. ${ }^{15}$ The use of autologous blood clot, viscoelastic devices, or perfluorocarbon liquid to prevent flap dislodgment during the air-fluid exchange has been investigated. $^{31,32}$ A novel technique of performing a semicircular single-layered ILM inverted flap assisted by sub-perfluorocarbon liquid injection of viscoelastic device provided good results, but the costs are increased. ${ }^{33}$ The utility of these adjuvants is not completely established. The spontaneous recovery of postoperative partial flap detachment from the retina may occur. ${ }^{34} \mathrm{In}$ addition, a method to evaluate the correct positioning of the flap with intraoperative real-time spectral-domain OCT is now available. ${ }^{35}$

We performed a comprehensive review of the scientific evidence on the anatomical and functional outcomes of the inverted ILM flap technique in the repair of macular holes, following the IVTS classification.

\section{Surgical Outcomes of Inverted ILM Flap Technique Full-Thickness Macular Holes (FTMH) By Size}

\section{Small FTMH $(\leq 250 \mu \mathrm{m})$}

There have been reports of spontaneous closure in FTMHs with the minimum linear diameter (MLD) below $250 \mu \mathrm{m} .{ }^{36,37}$ The spontaneous closure is accompanied by visual acuity (VA) improvement, but persistent defects in the ellipsoid zone and choriocapillaris were documented. ${ }^{37,38} \mathrm{~A}$ recent 
paper recognized the role of Muller cells in this rare phenomenon. ${ }^{39}$ The authors suggested that the treatment of small holes is not required if vision and hole size remains stable, and if the fovea of the fellow eye appears normal. However, the progressive enlargement of FTMH is frequent, and the rate of enlargement is accelerated in smaller holes. ${ }^{40}$ Besides, a better visual outcome is predicted by a smaller preoperative MLD which means that performing surgery in the early stage of an FTMH might improve the outcome. ${ }^{41}$

Nonsurgical procedures have been used with acceptable results. The IVI of ocriplasmin in small FTMHs with TVM has yielded a hole closure rate of $41-63 \%{ }^{42-44}$ Eyes with FTMHs $\leq 250 \mu \mathrm{m}$ are more likely to achieve nonsurgical closure after IVI of ocriplasmin. ${ }^{43}$ A recent case series reported a hole closure rate of $86 \%$ in FTMHs $\leq 250 \mu \mathrm{m}$ (with or without VMT) after IVI of C3F8 alone followed by face-down positioning, but the sample was small. ${ }^{45}$ The anatomical success increases when a surgical procedure is performed. Vitrectomy without ILM peeling had a hole closure rate varying between $55 \%$ and $100 \%$ in FTMHs $<400 \mu \mathrm{m}$ (stage 2 of Gass classification). ${ }^{46-48}$ Vitrectomy with ILM peeling and gas tamponade was associated with hole closure rate $>95 \%{ }^{49}$ The outcome of ILM peeling seems not to be dependent on the use of gas tamponade and face-down posturing. ${ }^{50}$ Vitrectomy, ILM peeling, and gas tamponade have shown efficacy in small FMTHs that failed to close after IVI of ocriplasmin. ${ }^{51}$ To our knowledge, no studies have reported the anatomical and functional results of the inverted ILM flap technique in FTMHs $\leq 250 \mu \mathrm{m}$. Instead, most studies used the cut-off of $400 \mu \mathrm{m}$ to differentiate between small to medium and large FTMHs. There are two possible reasons for this lack of evidence: ILM peeling is effective in almost all small FTMHs and this is the least common type of FTMHs. ${ }^{52}$

The appropriate management of small FTMHs is controversial. Small FTMHs observation is possible, but given the fact that a progressive enlargement is frequent, surgery either ILM peeling or inverted ILM flap technique could be a reasonable first-line approach. Additionally, the smaller is the diameter, the more chance to get a better visual outcome.

\section{Medium FTMH $(250 \mu \mathrm{m}-400 \mu \mathrm{m})$}

It is more consensual to perform surgery in medium FTMHs than in small FTMHs given the limited efficacy of nonsurgical procedures in FTMHs $>250 \mu \mathrm{m}$. The hole closure rate of IVI of ocriplasmin has been $34-36.8 \%$ for medium FTMHs. ${ }^{42,43}$ This percentage falls to $13 \%$ in the real-world setting. ${ }^{53}$ The hole closure rate of IVI of C3F8 alone followed by face-down positioning was $80 \%{ }^{45}$ Vitrectomy plus gas tamponade and face-down positioning resulted in hole closure in $100 \%$ of 18 cases. ${ }^{46}$ However, this procedure had a lower closure rate (55\%) in a randomized controlled trial (RCT), which led the authors to rather recommend ILM peeling to repair FTMHs. ${ }^{47}$ A meta-analysis including four RCTs concluded that ILM peeling yielded a higher anatomical success and reduced need for additional surgical intervention despite a similar long-term VA compared with non-ILM peeling. ${ }^{48}$ Liu et al reported a closure rate of $100 \%$ and a mean postoperative VA of $0.54 \log$ MAR at 12 months after vitrectomy with ILM peeling and gas tamponade. ${ }^{49}$ According to published data of a case series, good outcomes (closure rate of $85 \%$ ) are achieved after ILM peeling technique without the use of gas tamponade or postoperative positioning in small to medium FTMHs. ${ }^{50}$ A Cochrane review concluded that face-down positioning had no significant effect on successful hole closure in FTMHs $<400 \mu \mathrm{m}^{54}$ ILM peeling combined with air tamponade and non-supine positioning led to a closure rate of $95 \%$ in this type of FTMHs. ${ }^{55}$ In a large comparative study, the hole closure rate in FTMHs $<400 \mu$ m was $96 \%$ after ILM peeling, similar to $97 \%$ after inverted ILM flap technique, but postoperative VA was significantly better in those submitted to inverted ILM flap technique. ${ }^{16}$ No differences were found between inverted ILM flap and ILM flap technique regarding the postoperative VA and the integrity of the external retinal layers in FTMHs $<400 \mu \mathrm{m}$ in a recent work. ${ }^{56}$

The current literature does not support the use of the inverted ILM flap technique over ILM peeling in medium FTMHs. Both procedures are appropriate, and the option for one over the other should be based on the surgeon's preferences and experience.

\section{Large FTMH (>400 $\mu \mathrm{m})$}

In an epidemiologic study, large FTMHs had a prevalence of $55 \%$, followed by medium (31\%) and small (14\%) FTMHs. ${ }^{52}$ There are unsatisfactory results with nonsurgical procedures and vitrectomy without ILM peeling for large FTMHs. ${ }^{42,43,46,47,53,54}$ Liu et al reported a closure rate of $81 \%$ and a mean postoperative VA of $0.95 \log$ MAR after the combination of vitrectomy, ILM peeling, and gas tamponade. ${ }^{49}$ The use of air tamponade and non-supine positioning regimen is not effective in large $\mathrm{MHs}^{55}$ 
Facedown positioning provides a better visual outcome despite no apparent beneficial effect on anatomical success. $^{57}$ ILM peeling with autologous platelet-rich plasma on FTMH was superior to ILM peeling alone, with a hole closure rate of $100 \%$ in a total of 62 eyes. ${ }^{58}$ Initially, inverted ILM flap technique was proposed to improve the surgical outcomes for large FTMHs. ${ }^{88}$ There are extensive published data comparing ILM peeling and inverted ILM flap techniques. Two meta-analyses has demonstrated better anatomical and functional outcomes after inverted ILM flap technique versus ILM peeling in large FTMHs. ${ }^{59,60}$ In one of the comparative studies, the hole closure rate and VA improvement for FTMHs $>400 \mu \mathrm{m}$ were $95 \%$ and $75 \%$, respectively, after inverted ILM flap technique, and $87 \%$ and $57 \%$, respectively, after indocyanine green-assisted ILM peeling. ${ }^{61}$ Nevertheless, the differences in VA improvement between both techniques were more evident in the short term than in the long term. ${ }^{62}$ Regarding the recovery of normal retinal architecture, there is conflicting data. The restoration of foveal microstructure was more often observed 6 months after inverted ILM flap technique than after ILM peeling. ${ }^{63}$ Other studies have demonstrated lower postoperative recovery rate of the external limiting membrane (ELM) and ellipsoid zone (EZ) after inverted ILM flap (versus ILM peeling). ${ }^{64,65}$ In a total of 117 large idiopathic FTMHs, inverted ILM flap technique was associated with a higher closure rate, but the visual acuity and EZ integrity improved irrespective of the presence of an ILM flap. ${ }^{65}$ The complete ELM restoration is an important factor for VA improvement in the long term. ${ }^{66}$ Other studies compared inverted ILM flap technique with later surgical techniques. The free-flap technique had a closure rate of $86 \%$, compared to $92 \%$ of inverted ILM flap technique. ${ }^{67}$ The hole closure rate after the single-layer temporal inverted ILM flap technique was $100 \%{ }^{68}$ A study demonstrated a better visual result and a higher rate of hole closure after temporal inverted ILM flap technique versus classic inverted ILM flap technique, but further research is needed to confirm these results. ${ }^{69}$

The extra-large FTMHs have a MLD $>550-600 \mu \mathrm{m}$. In this subgroup, the anatomical success rates has been 3470\% and 63-90\%, after ILM peeling and inverted ILM flap technique, respectively. ${ }^{70,71}$ In 5 eyes with FTMHs $>1000 \mu \mathrm{m}, 4$ closed after inverted ILM flap technique with a visual improvement of approximately three lines. ${ }^{72}$ In a retrospective study that included FTMHs $>800 \mu \mathrm{m}$, the hole closure rate was $89 \%$ (versus $78 \%$ in ILM peeling group) accompanied by a higher gain in VA in the inverted ILM flap group. $^{73}$ A large Japanese study showed a closure rate of $100 \%$ in FTMHs either with MLD $\leq$ or $>550 \mu \mathrm{m}$ after inverted ILM flap technique, compared with $95 \%$ and $88 \%$ in FTMHs $\leq 550 \mu \mathrm{m}$ and $>550 \mu \mathrm{m}$, respectively, after ILM peeling. ${ }^{74}$

There is robust data that shows better anatomical and functional outcomes of inverted ILM flap technique in large and extra-large FTMHs compared with ILM peeling.

\section{By Vitreous Status}

Presence of VMT

VMT was estimated to be present in $34 \%$ of FTMHs, and in only $13 \%$ of small FTMHs. ${ }^{51,52}$ There is no relationship between the FTMH size and the presence of $\mathrm{VMT}^{75}$ The most relevant consideration in this type of FTMHs when they are small $(<250 \mu \mathrm{m})$ is the possibility of performing a nonsurgical technique, namely a IVI of ocriplasmin. The success rates of this procedure were mentioned above. When a nonsurgical procedure is not possible, vitrectomy regardless of the surgical technique allows for elimination of VMT.

\section{By Cause}

Primary FTMH (Idiopathic)

The idiopathic etiology is found in about $86 \%$ of FTMHs with a male-to-female ratio of $1: 2.2 .^{52}$ Vitrectomy for the treatment of FTMHs was first used in idiopathic and large holes. ${ }^{6}$ In this well-known work, $58 \%$ of the FTMHs closed using vitrectomy and gas tamponade. The anatomical success increased to over $85 \%$ since the ILM peeling and, later, the inverted ILM flap technique were introduced. In a comparative study, idiopathic FTMHs had better outcome after ILM peeling than secondary FTMHs. $^{76}$

As stated before, inverted ILM flap technique is the procedure of choice in large idiopathic FTMHs.

\section{Secondary FTMH}

The three main causes of secondary FTMHs are high myopia, diabetes mellitus, and ocular trauma.

The myopic FTHMs present with or without coexisting RD. In myopic FTMHs with RD, the hole closure and retinal reattachment rates have been $75-100 \%$ and $81-$ $100 \%$, respectively, after inverted ILM flap technique, and $25-55 \%$ and 55-93\%, respectively, after ILM peeling. ${ }^{77-79}$ Inverted ILM flap technique enabled a higher rate of retinal reattachment and closure in myopic FTMH-induced retinal detachment compared with ILM 
peeling, despite similar visual outcome. ${ }^{80}$ In another report, there was a better closure rate and postoperative VA in highly myopic eyes undergoing inverted ILM flap technique, but retinal reattachment rate was similar with both techniques. ${ }^{61}$ In myopic FTMHs without RD, the hole closure rate was $100 \%$ after inverted ILM flap procedure and $94 \%$ of the patients improved by at least 2 logMAR lines. ${ }^{81}$ Recent results from a meta-analysis has suggested that the inverted ILM flap technique is more effective regarding hole closure than ILM peeling in myopic FTMH with or without RD. ${ }^{60}$ The functional outcome of inverted ILM flap depend on the use of "cover" or "insertion" technique: VA improvement in 77\% (without $\mathrm{RD}$ ) and 95\% (with $\mathrm{RD}$ ) using "cover" technique; VA improvement in $66 \%$ (without $\mathrm{RD}$ ) and $80 \%$ (with RD) using "insertion" technique. ${ }^{82}$ Regarding the outer retina recovery, inverted ILM flap and ILM peeling techniques were no different in two studies. ${ }^{83,84} \mathrm{~A}$ third comparative study showed a higher rate of ELM and EZ recovery after inverted ILM flap technique. ${ }^{85}$ The use of perfluorocarbon liquid in FTMHs with RD and viscoat ${ }^{\circledR}$ in FTMHs without RD combined with inverted ILM flap technique were proposed to increase the success rate of the surgery. ${ }^{86,87}$

Comparing with idiopathic FTMHs, traumatic FTMHs have a higher likelihood of spontaneous closure (up to $67 \%$ ) that often occurs within 3 months after ocular trauma ${ }^{88}$ There was a trend toward small size in spontaneously closed traumatic FTMHs. ${ }^{89}$ Vitrectomy regardless of the surgical technique results in good anatomical and visual results in these cases. ${ }^{90}$ ILM peeling resulted in $100 \%$ closure rate, with vision improving by at least 2 Snellen lines in $94 \%$ of 17 consecutive eyes with traumatic FTMH. ${ }^{91}$ This technique was also shown to be effective in pediatric traumatic FTMHs. $^{92}$ Both gas or silicone oil tamponades can be used. ${ }^{93}$ Inverted ILM flap technique has been evaluated in large traumatic FTMH which commonly are closed after this technique. ${ }^{94-96}$ In a case series of 12 large traumatic FTMHs, all closed after the inverted ILM flap technique. ${ }^{88}$ Recently, it was described a case in which inverted ILM flap assisted by autologous plasma concentrate was successfully performed in a pediatric patient with traumatic large $\mathrm{FTMH}^{97} \mathrm{~A}$ comparative study showed a closure rate of $92 \%$ and $75 \%$, and VA improvement of 5 lines and 2.5 lines after inverted ILM flap and ILM peeling, respectively. ${ }^{98}$ The authors concluded that the inverted ILM flap technique provides a better outcome in traumatic FTMHs than ILM peeling.
In eyes with concomitant FTMH and proliferative diabetic retinopathy (PDR), the hole closure was observed in $83 \%$ after vitrectomy regardless the use of ILM peeling. ${ }^{99}$ In a study that included eyes with non-proliferative diabetic retinopathy and PDR, the hole closure rate of inverted ILM flap technique was $84 \% .^{100}$ The authors speculated that diabetic FTMHs have a different morphology and healing process compared with idiopathic cases which compromises the prognosis. In a case series of eyes with PDR and coexisting retinal detachment, all closed after inverted ILM flap technique or free ILM flap. ${ }^{101}$

A rare case of secondary FTMH developing after antiangiogenic intravitreal injection for neovascular AMD in which temporal inverted ILM flap technique allowed for the resolution of the macular hole was reported. ${ }^{102}$

According to the literature, the inverted ILM flap technique is the most effective procedure in secondary FTMHs, particularly in myopic and traumatic FTMHs.

\section{Other Potential Indications of Inverted ILM Flap Technique}

Frisina et al proposed a technique similar to inverted ILM flap procedure to treat idiopathic LMHs. ${ }^{103}$ The authors reported that the double inverted ERM and ILM flap yield a better functional prognosis and reduce the iatrogenic damage of the fovea or the risk of inducing postoperative FTMH.

\section{Conclusion}

Answering the question in the title of this paper, inverted ILM flap technique is the best option for primary surgical approach of large idiopathic FTMHs, and secondary FTMHs. Other techniques have been introduced but, at the data of this comprehensive review of the literature, the evidence is scarce to recommend its widespread use.

\section{Disclosure}

The authors report no conflicts of interest for this work.

\section{References}

1. Gass JD. Idiopathic senile macular hole. Its early stages and pathogenesis. Arch Ophthalmol. 1988;106(5):629-639. doi:10.1001/ archopht.1988.01060130683026

2. Duker JS, Kaiser PK, Binder S, et al. The International Vitreomacular Traction Study Group classification of vitreomacular adhesion, traction, and macular hole. Ophthalmology. 2013;120(12):2611-2619. doi:10.1016/j.ophtha.2013.07.042

3. Gattoussi S, Buitendijk GHS, Peto T, et al. The European eye epidemiology spectral-domain optical coherence tomography classification of macular diseases for epidemiological studies. Acta Ophthalmol. 2019;97(4):364-371. doi:10.1111/aos.13883 
4. Gass JDM, editor. Stereoscopic atlas of macular diseases: Diagnosis and Treatment. 1997:158-165.

5. Johnson RN, Gass JD. Idiopathic macular holes. Observations, stages of formation, and implications for surgical intervention. Ophthalmology. 1988;95(7):917-924. doi:10.1016/S01616420(88)33075-7

6. Kelly NE, Wendel RT. Vitreous surgery for idiopathic macular holes. Results of a pilot study. Arch Ophthalmol. 1991;109 (5):654-659. doi:10.1001/archopht.1991.01080050068031

7. Park DW, Sipperley JO, Sneed SR, Dugel PU, Jacobsen J. Macular hole surgery with internal-limiting membrane peeling and intravitreous air. Ophthalmology. 1999;106(7):1392-1398. doi:10.1016/S0161-6420(99)00730-7

8. Michalewska Z, Michalewski J, Adelman RA, Nawrocki J. Inverted internal limiting membrane flap technique for large macular holes. Ophthalmology. 2010;117(10):2018-2025. doi:10.1016/j.ophtha.2010.02.011

9. Mahajan VB, Chin EK, Tarantola RM, et al. Macular hole closure with internal limiting membrane abrasion technique. JAMA Ophthalmol. 2015;133(6):635-641. doi:10.1001/ jamaophthalmol.2015.204

10. Wolfensberger TJ. Autologous Internal Limiting Membrane (ILM) recycling to close very large refractory macular holes. Klin Monbl Augenheilkd. 2015;232(4):585-586. doi:10.1055/ s-0035-1545681

11. Pichi F, Abboud EB. Spare some internal limiting membrane for later: free ILM patch and neurosensory retina graft. Int Ophthalmol. 2019;39(5):1205-1207. doi:10.1007/s10792-018-0906-2

12. Rizzo S, Caporossi T, Tartaro R, et al. A human amniotic membrane plug to promote retinal breaks repair and recurrent macular hole closure. Retina. 2019;39(Suppl 1):S95-S103. doi:10.1097/ IAE.0000000000002320

13. Cisiecki S, Boninska K, Bednarski M, Rizzo S. Autologous lens capsule flap transplantation for persistent macular holes. J Ophthalmol. 2021;2021:8148792. doi:10.1155/2021/8148792

14. Moisseiev J, Moroz I, Katz G. Effect of ocriplasmin on the management of macular holes: assessment of the clinical relevance of ocriplasmin. JAMA Ophthalmol. 2014;132(6):709-713. doi:10.1001/jamaophthalmol.2013.8223

15. Silva N, Ferreira N, Pessoa B, Correia N, Beirao JM, Meireles A. Inverted internal limiting membrane flap technique in the surgical treatment of macular holes: 8-year experience. Int Ophthalmol. 2021;41(2):499-507. doi:10.1007/s10792-020-01600-4

16. Rizzo S, Tartaro R, Barca F, Caporossi T, Bacherini D, Giansanti F. Internal limiting membrane peeling versus inverted flap technique for treatment of full-thickness macular holes: a Comparative Study in a large series of patients. Retina. 2018;38(Suppl 1):S73-S78. doi:10.1097/IAE.0000000000001985

17. Shiode Y, Morizane Y, Matoba R, et al. The role of inverted internal limiting membrane flap in macular hole closure. Invest Ophthalmol Vis Sci. 2017;58(11):4847-4855. doi:10.1167/ iovs.17-21756

18. Boninska K, Nawrocki J, Michalewska Z. Mechanism of "flap closure" after the inverted internal limiting membrane flap technique. Retina. 2018;38(11):2184-2189. doi:10.1097/ IAE.0000000000001861

19. Michalewska Z, Michalewski J, Dulczewska-Cichecka K, Adelman RA, Nawrocki J. Temporal inverted internal limiting membrane flap technique versus classic inverted internal limiting membrane flap technique: a Comparative Study. Retina. 2015;35 (9):1844-1850. doi:10.1097/IAE.0000000000000555

20. Ghassemi F, Khojasteh H, Khodabande A, et al. Comparison of three different techniques of inverted internal limiting membrane flap in treatment of large idiopathic full-thickness macular hole. Clin Ophthalmol. 2019;13:2599-2606. doi:10.2147/OPTH. S236169
21. $\mathrm{Hu} \mathrm{Z}$, Qian H, Fransisca $\mathrm{S}$, et al. Minimal internal limiting membrane peeling with ILM flap technique for idiopathic macular holes: a preliminary study. BMC Ophthalmol. 2020;20(1):228. doi:10.1186/s12886-020-01505-x

22. Kaluzny JJ, Zabel P, Kaluzna M, et al. Macular sensitivity in area of internal limiting membrane (ILM) peeling in eyes after pars plana vitrectomy with temporal inverted ILM flap technique for full thickness macular hole. Retina. 2021;41:1627-1634. doi:10.1097/IAE.0000000000003096

23. Avci R, Mavi Yildiz A, Yilmaz S. Evaluation of inner retinal dimples and internal limiting membrane flap configuration after temporal inverted ILM flap technique. Eur $J$ Ophthalmol. 2021;31(2):649-655. doi:10.1177/1120672120940980

24. Karalezli A, Kaderli ST, Sul S. Macular microvasculature differences in patients with macular hole after vitrectomy with internal limiting membrane removal or single-layered temporal inverted flap technique. Eye (Lond). 2020. doi:10.1038/s41433-020-01301-3

25. Aurora A, Seth A, Sanduja N. Cabbage leaf inverted flap ILM peeling for macular hole: a novel technique. Ophthalmic Surg Lasers Imaging Retina. 2017;48(10):830-832. doi:10.3928/ 23258160-20170928-08

26. Faria MY, Proenca H, Ferreira NG, Sousa DC, Neto E, MarquesNeves C. Inverted internal limiting membrane flap techniques and outer retinal layer structures. Retina. 2020;40(7):1299-1305. doi:10.1097/IAE.0000000000002607

27. Cacciamani A, Gelso A, Di Nicola M, et al. Inverted ILM-flap techniques variants for macular hole surgery: randomized clinical trial to compare retinal sensitivity and fixation stability. Sci Rep. 2020;10(1):15832. doi:10.1038/s41598-020-72774-1

28. Iwasaki M, Miyamoto $\mathrm{H}$, Imaizumi $\mathrm{H}$. Effects of inverted internal limiting membrane technique and insertion technique on outer retinal restoration associated with glial proliferation in large macular holes. Graefes Arch Clin Exp Ophthalmol. 2020;258 (9):1841-1849. doi:10.1007/s00417-020-04655-2

29. Park JH, Lee SM, Park SW, Lee JE, Byon IS. Comparative analysis of large macular hole surgery using an internal limiting membrane insertion versus inverted flap technique. $\mathrm{Br}$ J Ophthalmol. 2019;103(2):245-250. doi:10.1136/bjophthalmol2017-311770

30. Zhu K, Lei B, Wong W, et al. Comparison of the internal limiting membrane insertion technique and the inverted internal limiting membrane flap technique with vitrectomy to treat macular hole-associated retinal detachment. Retina. 2021;41(1):37-44. doi:10.1097/IAE.0000000000002804

31. Andrew N, Chan WO, Tan M, Ebneter A, Gilhotra JS. Modification of the inverted internal limiting membrane flap technique for the treatment of chronic and large macular holes. Retina. 2016;36(4):834-837. doi:10.1097/IAE.000000000 0000931

32. Shin MK, Park KH, Park SW, Byon IS, Lee JE. Perfluoron-octane-assisted single-layered inverted internal limiting membrane flap technique for macular hole surgery. Retina. 2014;34 (9):1905-1910. doi:10.1097/IAE.0000000000000339

33. Chou HD, Chong YJ, Teh WM, et al. Nasal or temporal internal limiting membrane flap assisted by sub-perfluorocarbon viscoelastic injection for macular hole repair. Am $J$ Ophthalmol. 2021;223:296-305. doi:10.1016/j.ajo.2020.09.023

34. Kawamata Y, Baba T, Yokouchi H, Yamamoto S. Partial detachment of internal limiting membrane flap and spontaneous re-covering of macular hole by flap. Am J Ophthalmol Case Rep. 2021;22:101089. doi:10.1016/j.ajoc.2021.101089

35. Lytvynchuk LM, Falkner-Radler CI, Krepler K, et al. Dynamic intraoperative optical coherence tomography for inverted internal limiting membrane flap technique in large macular hole surgery. Graefes Arch Clin Exp Ophthalmol. 2019;257(8):1649-1659. doi:10.1007/s00417-019-04364-5 
36. Tadayoni R, Massin P, Haouchine B, Cohen D, Erginay A, Gaudric A. Spontaneous resolution of small stage 3 and 4 full-thickness macular holes viewed by optical coherence tomography. Retina. 2001;21(2):186-189. doi:10.1097/ 00006982-200104000-00019

37. Privat E, Tadayoni R, Gaucher D, Haouchine B, Massin P, Gaudric A. Residual defect in the foveal photoreceptor layer detected by optical coherence tomography in eyes with spontaneously closed macular holes. Am J Ophthalmol. 2007;143 (5):814-819. doi:10.1016/j.ajo.2006.12.039

38. Nawrocka ZA, Nawrocka Z, Nawrocki J. Spontaneous closure of full-thickness macular holes. Ophthalmologica. 2021. doi:10.1159/000516768

39. Bringmann A, Duncker T, Jochmann C, Barth T, Duncker GIW, Wiedemann P. Spontaneous closure of small full-thickness macular holes: presumed role of Muller cells. Acta Ophthalmol. 2020;98(4):e447-e456. doi:10.1111/aos.14289

40. Berton M, Robins J, Frigo AC, Wong R. Rate of progression of idiopathic full-thickness macular holes before surgery. Eye. 2020;34(8):1386-1391. doi:10.1038/s41433-019-0654-1

41. Fallico M, Jackson TL, Chronopoulos A, et al. Factors predicting normal visual acuity following anatomically successful macular hole surgery. Acta Ophthalmol. 2021;99(3):e324-e329. doi:10.1111/aos.14575

42. Dugel PU, Tolentino M, Feiner L, Kozma P, Leroy A. Results of the 2-year ocriplasmin for treatment for symptomatic vitreomacular adhesion including macular hole (OASIS) randomized trial. Ophthalmology. 2016;123(10):2232-2247. doi:10.1016/j. ophtha.2016.06.043

43. Haller JA, Stalmans P, Benz MS, et al. Efficacy of intravitreal ocriplasmin for treatment of vitreomacular adhesion: subgroup analyses from two randomized trials. Ophthalmology. 2015;122 (1):117-122. doi:10.1016/j.ophtha.2014.07.045

44. Figueira J, Martins D, Pessoa B, et al. The Portuguese experience with ocriplasmin in clinical practice. Ophthalmic Res. 2016;56 (4):186-192. doi:10.1159/000446842

45. Han R, Zhang C, Zhao X, Chen Y. Treatment of primary full-thickness macular hole by intravitreal injection of expansile gas. Eye. 2019;33(1):136-143. doi:10.1038/s41433-018-0098-z

46. Tadayoni R, Gaudric A, Haouchine B, Massin P. Relationship between macular hole size and the potential benefit of internal limiting membrane peeling. $\mathrm{Br} J$ Ophthalmol. 2006;90 (10):1239-1241. doi:10.1136/bjo.2006.091777

47. Christensen UC, Kroyer K, Sander B, et al. Value of internal limiting membrane peeling in surgery for idiopathic macular hole stage 2 and 3: a randomised clinical trial. Br J Ophthalmol. 2009;93(8):1005-1015. doi:10.1136/bjo.2008.151266

48. Spiteri Cornish K, Lois N, Scott NW, et al. Vitrectomy with internal limiting membrane peeling versus no peeling for idiopathic full-thickness macular hole. Ophthalmology. 2014;121 (3):649-655. doi:10.1016/j.ophtha.2013.10.020

49. Liu L, Enkh-Amgalan I, Wang NK, et al. Results of macular hole surgery: evaluation based on the International Vitreomacular Traction Study classification. Retina. 2018;38(5):900-906. doi:10.1097/IAE.0000000000001647

50. Lally DR, Kasetty MA. Closure of small macular holes using vitrectomy surgery with internal limiting membrane peeling without the use of intraocular gas tamponade: broadening the understanding of the macular hole pathophysiology. Retin Cases Brief Rep. 2020;14(2):104-109. doi:10.1097/ICB.0000000000000919

51. Benarous A, Le Mer Y. Long-term results of vitrectomy for macular holes after failure of vitreolysis. Ophthalmologica. 2018;240(1):14-22. doi:10.1159/000481893

52. Forsaa VA, Lindtjorn B, Kvaloy JT, Froystein T, Krohn J. Epidemiology and morphology of full-thickness macular holes. Acta Ophthalmol. 2018;96(4):397-404. doi:10.1111/aos.13618
53. Schumann RG, Langer J, Compera D, et al. Assessment of intravitreal ocriplasmin treatment for vitreomacular traction in clinical practice. Graefes Arch Clin Exp Ophthalmol. 2017;255 (11):2081-2089. doi:10.1007/s00417-017-3747-1

54. Solebo AL, Lange CA, Bunce C, Bainbridge JW. Face-down positioning or posturing after macular hole surgery. Cochrane Database Syst Rev. 2011;12:CD008228.

55. Forsaa VA, Krohn J. Air tamponade combined with nonsupine positioning in macular hole surgery for pseudophakic eyes. Retina. 2017;37(9):1750-1756. doi:10.1097/IAE.000000000 0001413

56. Baumann C, Dervenis N, Kirchmair K, Lohmann CP, Kaye SB, Sandinha MT. Functional and morphological outcomes of the inverted internal limiting membrane flap technique in small and medium sized macular holes <400microm. Retina. 2021.

57. Pasu S, Bell L, Zenasni Z, et al. Facedown positioning following surgery for large full-thickness macular hole: a multicenter randomized clinical trial. JAMA Ophthalmol. 2020;138(7):725-730. doi:10.1001/jamaophthalmol.2020.0987

58. Shpak AA, Shkvorchenko DO, Krupina EA. Surgical treatment of macular holes with and without the use of autologous platelet-rich plasma. Int Ophthalmol. 2021;41(3):1043-1052. doi:10.1007/ s10792-020-01662-4

59. Chen G, Tzekov R, Jiang F, Mao S, Tong Y, Li W. Inverted ILM flap technique versus conventional ILM peeling for idiopathic large macular holes: a meta-analysis of randomized controlled trials. PLoS One. 2020;15(7):e0236431. doi:10.1371/journal. pone. 0236431

60. Marques RE, Sousa DC, Leal I, Faria MY, Marques-Neves C. Complete ILM peeling versus inverted flap technique for macular hole surgery: a meta-analysis. Ophthalmic Surg Lasers Imaging Retina. 2020;51(3):187-A182. doi:10.3928/23258160-2020022808

61. Gu C, Qiu Q. Inverted internal limiting membrane flap technique for large macular holes: a systematic review and single-arm meta-analysis. Graefes Arch Clin Exp Ophthalmol. 2018;256 (6):1041-1049. doi:10.1007/s00417-018-3956-2

62. Shen Y, Lin X, Zhang L, Wu M. Comparative efficacy evaluation of inverted internal limiting membrane flap technique and internal limiting membrane peeling in large macular holes: a systematic review and meta-analysis. BMC Ophthalmol. 2020;20(1):14. doi:10.1186/s12886-019-1271-2

63. Ramtohul P, Parrat E, Denis D, Lorenzi U. Inverted internal limiting membrane flap technique versus complete internal limiting membrane peeling in large macular hole surgery: a comparative study. BMC Ophthalmol. 2020;20(1):11. doi:10.1186/s12886-019-1294-8

64. Iwasaki M, Kinoshita T, Miyamoto H, Imaizumi H. Influence of inverted internal limiting membrane flap technique on the outer retinal layer structures after a large macular hole surgery. Retina. 2019;39(8):1470-1477. doi:10.1097/IAE.0000000000002209

65. Baumann C, Kaye S, Iannetta D, Sultan Z, Dwivedi R, Pearce I. Effect of inverted internal limiting membrane flap on closure rate, postoperative visual acuity, and restoration of outer retinal layers in primary idiopathic macular hole surgery. Retina. 2020;40 (10):1955-1963. doi:10.1097/IAE.0000000000002707

66. Bleidissel N, Friedrich J, Klaas J, Feucht N, Lohmann CP, Maier M. Inverted internal limiting membrane flap technique in eyes with large idiopathic full-thickness macular hole: long-term functional and morphological outcomes. Graefes Arch Clin Exp Ophthalmol. 2021;259:1-13.

67. Velez-Montoya R, Ramirez-Estudillo JA, Sjoholm-gomez de Liano C, et al. Inverted ILM flap, free ILM flap and conventional ILM peeling for large macular holes. Int J Retina Vitr. 2018;4 (1):8. doi:10.1186/s40942-018-0111-5 
68. Takai Y, Tanito M, Sugihara K, Ohira A. The role of single-layered flap in temporal inverted internal limiting membrane flap technique for macular holes: pros and cons. J Ophthalmol. 2019;2019:5737083. doi:10.1155/2019/5737083

69. Avci R, Mavi Yildiz A, Yilmaz S. Conventional internal limiting membrane peeling versus temporal inverted internal limiting membrane flap for large macular holes. Eur J Ophthalmol. 2021;31(2):679-687. doi:10.1177/1120672119892427

70. Kannan NB, Kohli P, Parida H, Adenuga OO, Ramasamy K. Comparative study of inverted internal limiting membrane (ILM) flap and ILM peeling technique in large macular holes: a randomized-control trial. BMC Ophthalmol. 2018;18(1):177. doi:10.1186/s12886-018-0826-y

71. Manasa S, Kakkar P, Kumar A, Chandra P, Kumar V, Ravani R. Comparative evaluation of standard ILM peel with inverted ILM flap technique in large macular holes: a prospective, Randomized Study. Ophthalmic Surg Lasers Imaging Retina. 2018;49 (4):236-240. doi:10.3928/23258160-20180329-04

72. Khodani M, Bansal P, Narayanan R, Chhablani J. Inverted internal limiting membrane flap technique for very large macular hole. Int J Ophthalmol. 2016;9(8):1230-1232.

73. Narayanan R, Singh SR, Taylor S, et al. Surgical outcomes after inverted internal limiting membrane flap versus conventional peeling for very large macular holes. Retina. 2019;39 (8):1465-1469. doi:10.1097/IAE.0000000000002186

74. Yamashita T, Sakamoto T, Terasaki H, et al. Best surgical technique and outcomes for large macular holes: retrospective multicentre study in Japan. Acta Ophthalmol. 2018;96(8):e904-e910. doi:10.1111/aos.13795

75. Philippakis E, Amouyal F, Couturier A, Boulanger-Scemama E, Gaudric A, Tadayoni R. Size and vitreomacular attachment of primary full-thickness macular holes. Br J Ophthalmol. 2017;101 (7):951-954. doi:10.1136/bjophthalmol-2016-309212

76. Ghoraba HH, Leila M, Zaky AG, et al. Results of pars plana vitrectomy for different types of macular holes. Clin Ophthalmol. 2021;15:551-557. doi:10.2147/OPTH.S290404

77. Takahashi H, Inoue M, Koto $\mathrm{T}$, Itoh $\mathrm{Y}$, Hirota $\mathrm{K}$, Hirakata A. Inverted internal limiting membrane flap technique for treatment of macular hole retinal detachment in highly myopic eyes. Retina. 2018;38(12):2317-2326. doi:10.1097/IAE.000000 0000001898

78. Sasaki H, Shiono A, Kogo J, et al. Inverted internal limiting membrane flap technique as a useful procedure for macular hole-associated retinal detachment in highly myopic eyes. Eye. 2017;31(4):545-550. doi:10.1038/eye.2016.263

79. Matsumae H, Morizane Y, Yamane S, et al. Inverted internal limiting membrane flap versus internal limiting membrane peeling for macular hole retinal detachment in high myopia. Ophthalmol Retina. 2020;4(9):919-926. doi:10.1016/j. oret.2020.03.021

80. Yuan J, Zhang LL, Lu YJ, Han MY, Yu AH, Cai XJ. Vitrectomy with internal limiting membrane peeling versus inverted internal limiting membrane flap technique for macular hole-induced retinal detachment: a systematic review of literature and meta-analysis. BMC Ophthalmol. 2017;17(1):219. doi:10.1186/ s12886-017-0619-8

81. Michalewska Z, Michalewski J, Dulczewska-Cichecka K, Nawrocki J. Inverted internal limiting membrane flap technique for surgical repair of myopic macular holes. Retina. 2014;34 (4):664-669. doi:10.1097/IAE.0000000000000042

82. Chatziralli I, Machairoudia G, Kazantzis D, Theodossiadis G, Theodossiadis P. Inverted internal limiting membrane flap technique for myopic macular hole: a meta-analysis. Surv Ophthalmol. 2021;66(5):771-780. doi:10.1016/j. survophthal.2021.02.010
83. Mete M, Alfano A, Maggio E, Guerriero M, Pertile G. Inverted ILM flap for the treatment of myopic macular holes: healing processes and morphological changes in comparison with complete ILM removal. $J$ Ophthalmol. 2019;2019:1314989. doi:10.1155/2019/1314989

84. Bove Alvarez M, Sabate S, Gomez-Resa M, Garcia-Arumi J. Anatomical and visual outcomes of inverted internal limiting membrane flap technique versus internal limiting membrane peeling in myopic macular hole without retinal detachment: a preliminary Retrospective Study. Retina. 2020;40(2):233-240. doi:10.1097/IAE.0000000000002368

85. Hu XT, Pan QT, Zheng JW, Zhang ZD. Foveal microstructure and visual outcomes of myopic macular hole surgery with or without the inverted internal limiting membrane flap technique. $\mathrm{Br}$ J Ophthalmol. 2019;103(10):1495-1502. doi:10.1136/bjophthalmol-2018-313311

86. Gu X, Hu Z, Qian H, et al. Perfluorocarbon liquid-assisted inverted internal limiting membrane flap technique versus internal limiting membrane peeling for highly myopic macular hole retinal detachment. Retina. 2021;41(2):317-323. doi:10.1097/ IAE.0000000000002853

87. Song Z, Li M, Liu J, Hu X, Hu Z, Chen D. Viscoat assisted inverted internal limiting membrane flap technique for large macular holes associated with high myopia. J Ophthalmol. 2016;2016:8283062. doi:10.1155/2016/8283062

88. Liu W, Grzybowski A. Current management of traumatic macular holes. J Ophthalmol. 2017;2017:1748135. doi:10.1155/2017/ 1748135

89. Miller JB, Yonekawa Y, Eliott D, et al. Long-term follow-up and outcomes in traumatic macular holes. Am J Ophthalmol. 2015;160(6):1255-1258 e1251. doi:10.1016/j.ajo.2015.09.004

90. Tang YF, Chang A, Campbell WG, et al. Surgical management of traumatic macular hole: optical coherence tomography features and outcomes. Retina. 2020;40(2):290-298. doi:10.1097/ IAE.0000000000002382

91. Kuhn F, Morris R, Mester V, Witherspoon CD. Internal limiting membrane removal for traumatic macular holes. Ophthalmic Surg Lasers. 2001;32(4):308-315. doi:10.3928/1542-8877-2001070109

92. Azevedo S, Ferreira N, Meireles A. Management of pediatric traumatic macular holes - case report. Case Rep Ophthalmol. 2013;4(2):20-27. doi:10.1159/000350543

93. Bor'i A, Al-Aswad MA, Saad AA, Hamada D, Mahrous A. Pars plana vitrectomy with internal limiting membrane peeling in traumatic macular hole: $14 \%$ perfluoropropane $(\mathrm{C} 3 \mathrm{~F} 8)$ versus silicone oil tamponade. J Ophthalmol. 2017;2017:3917696. doi:10.1155/2017/3917696

94. Astir S, Shroff D, Gupta C, Shroff C. Inverted flap technique for a large traumatic macular hole with choroidal rupture and subretinal hemorrhage. Oman J Ophthalmol. 2018;11 (1):68-70

95. Kumari A, Agarwal L, Agrawal N, Sahu S, Prasad I, Pradhan D. Inverted temporal internal limiting membrane flap technique for chronic large traumatic macular hole. GMS Ophthalmol Cases. 2020;10:Doc27.

96. Browne AW, Conti FF, Kaiser PK, Singh RP. Surgical repair of traumatic macular holes with extreme macular pathology using an internal limiting membrane flap technique. Ophthalmic Surg Lasers Imaging Retina. 2018;49(10):e143-e146. doi:10.3928/ 23258160-20181002-17

97. Finn AP, Chen X, Viehland C, Izatt JA, Toth CA, Vajzovic L. Combined internal limiting membrane flap and autologous plasma concentrate to close a large traumatic macular hole in a pediatric patient. Retin Cases Brief Rep. 2021;15(2):107-109. doi:10.1097/ ICB.0000000000000762 
98. Ghoraba HH, Leila M, Ghoraba H, Heikal MA, Elgemai EEM. Comparative Study between pars plana vitrectomy with internal limiting membrane peel and pars plana vitrectomy with internal limiting membrane flap technique for management of traumatic full thickness macular holes. J Ophthalmol. 2019;2019:1959082. doi:10.1155/2019/1959082

99. Yeh PT, Cheng CK, Chen MS, Yang CH, Yang CM. Macular hole in proliferative diabetic retinopathy with fibrovascular proliferation. Retina. 2009;29(3):355-361. doi:10.1097/ IAE.0b013e31818c3251

100. Michalewska Z, Nawrocki J. Fovea morphology after vitrectomy in eyes with full-thickness macular hole coexisting with diabetic retinopathy. J Vitreoretin Dis. 2021;5(1):53-59. doi:10.1177/ 2474126420944048
101. Chen SN, Yang CM. Internal limiting membrane flaps for coexistent macular hole and retinal detachment in eyes with proliferative diabetic retinopathy. $J$ Ophthalmol. 2018;2018:3470731. doi:10.1155/2018/3470731

102. Nawrocka ZA, Nawrocka Z, Nawrocki J. Vitrectomy for full thickness macular hole developed during the course of anti-VEGF treatment of type 1 neovascular AMD. Eur J Ophthalmol. 2021;11206721211002123.

103. Frisina R, Parrozzani R, Pilotto E, Midena E. A double inverted flap surgical technique for the treatment of idiopathic lamellar macular hole associated with atypical epiretinal membrane. Ophthalmologica. 2019;242(1):49-58. doi:10.1159/000496297

\section{Publish your work in this journal}

Clinical Ophthalmology is an international, peer-reviewed journal covering all subspecialties within ophthalmology. Key topics include: Optometry; Visual science; Pharmacology and drug therapy in eye diseases; Basic Sciences; Primary and Secondary eye care; Patient Safety and Quality of Care Improvements. This journal is indexed on PubMed

Submit your manuscript here: https://www.dovepress.com/clinical-ophthalmology-journal
Central and CAS, and is the official journal of The Society of Clinical Ophthalmology (SCO). The manuscript management system is completely online and includes a very quick and fair peer-review system, which is all easy to use. Visit http://www.dovepress.com/ testimonials.php to read real quotes from published authors. 